\title{
3D Freehand Ultrasound Reconstruction Based on Probe Trajectory
}

\author{
Pierrick Coupé, Pierre Hellier, Noura Azzabou, and Christian Barillot \\ Project VisAGeS, IRISA - INRIA - INSERM, \\ IRISA campus Beaulieu 35042 Rennes Cedex, France \\ \{pcoupe, phellier, cbarillo\}@irisa.fr, azzabou@certis.enpc.fr \\ http://www.irisa.fr/visages
}

\begin{abstract}
D freehand ultrasound imaging is a very attractive technique in medical examinations and intra-operative stage for its cost and field of view capacities. This technique produces a set of non parallel B-scans which are irregularly distributed in the space. Reconstruction amounts to computing a regular lattice volume and is needed to apply conventional computer vision algorithms like registration. In this paper, a new 3D reconstruction method is presented, taking explicitly into account the probe trajectory. Experiments were conducted on different data sets with various probe motion types and indicate that this technique outperforms classical methods, especially on low acquisition frame rate.
\end{abstract}

\section{Introduction}

Due to its low cost, real time image formation capability and non invasive nature, $2 \mathrm{D}$ ultrasound is a popular medical imaging modality. Nevertheless, the lack of $3 \mathrm{D}$ information prevents reproductivity, longitudinal follow-up and precise quantitative measurements. 3D ultrasound imaging addresses these disadvantages in order to obtain an objective representation of the scanned volume. Among the various options to acquire 3D ultrasound, this work focuses on 3D freehand ultrasound. This technique consists in tracking in the space a standard $2 \mathrm{D}$ probe by using 3D localizer (magnetic or optic). The tracking system continuously measures the 3D position and orientation of the probe. Contrary to mechanical built-in probes, freehand imaging is cheap, can address a large variety of clinical applications and allows large organs examination. However, the reconstruction step is still an acute problem with regards to computation time and reconstruction quality.

The sparsity of data is the main difficulty to transform a non uniformly distributed set of B-scans into a regular 3D volume. A correct reconstruction should not introduce geometrical artifacts, degrade nor distort the images. The most common techniques to resolve this problem are pixel nearest-neighbor (PNN) [3, voxel nearest-neighbor (VNN) [6] and distance-weighted (DW) interpolation [1,8. These approaches are designed to reduce computation time, but lead to a moderate reconstruction quality. More elaborated and recent methods 
build on non rigid registration [2], radial basis functions (RBF) interpolation [4] or Rayleigh model for intensity distribution [5]. Quality improvement is obtained at the expense of computational burden.

Another strategy in 3D freehand imaging consists in analyzing the 3D volume without reconstruction. The StradX system [3] is built on this approach. The sequence of B-scans can be arbitrarily resliced and distance/volume measurements are performed without reconstruction. This strategy is very powerful for manual analysis of 3D datasets. However, we do think that 3D isotropic reconstruction is still necessary for specific clinical context, especially when automatic segmentation or registration procedures are required.

This paper is organized as follows. Section 2 describes the proposed reconstruction method based on utilization of the probe trajectory (PT) information. Section 3 describes briefly the evaluation framework and compare the proposed method with pixel nearest-neighbor (PNN) and distance-weighted (DW) interpolation techniques.

\section{Method}

This work builds on the distance weighted (DW) interpolation and proposes to incorporate probe trajectory information. The DW interpolation is first presented in section (2.1). Then the probe trajectory information is incorporated in section (2.2).

\subsection{Distance Weighted (DW) Interpolation}

At each point $X$ of the reconstructed volume, the linear interpolation amounts to computing:

$$
f_{n}(X)=\frac{1}{G} \sum_{i \in K_{n}(X)} g_{i} \tilde{f}\left(X_{i}\right)
$$

where $K_{n}$ is the interpolation kernel. In other words, $K_{n}$ is the set of the different indexes of the B-scans that are involved in the interpolation. $n$ is the interpolation order. For a given interpolation degree, the $n$ closest B-scans before $X$ and the $n$ closest B-scans after $X$ are considered. For the DW interpolation, $X_{i}$ is the orthogonal projected of $X$ on the $i^{t h}$ B-scan. $\tilde{f}\left(X_{i}\right)$ is the intensity on the position $X_{i}$ and is obtained by a bilinear interpolation. Finally, $G$ is the normalization constant with $G=\sum g_{i}$, and $g_{i}$ is the distance between $X$ and $X_{i}$ (see Fig. 1). It might happen that a part of the reconstructed volume is visible at different time stamp (or view points) of the B-scans sequence. This is also known as spatial compounding. These different time stamps are computed so as to track this information and fully exploit the speckle decorrelation.

\subsection{Probe Trajectory}

Contrary to the classical DW interpolation approach where orthogonal projections are performed, the set of points $X_{i}$ is defined as the intersection between 


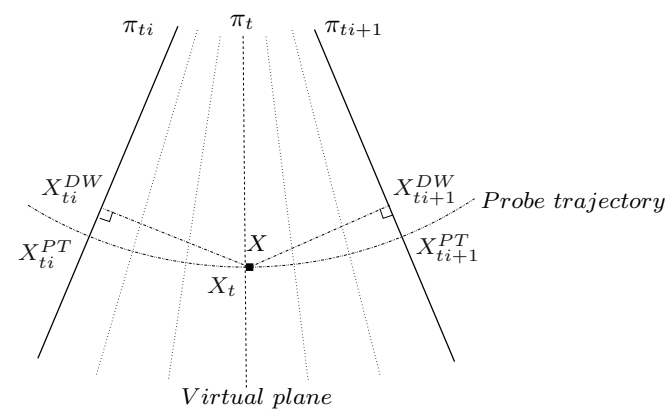

Fig. 1. Illustration of DW and PT principles. The two orthogonal projections for DW interpolation method and the construction of a "virtual" plane $\pi_{t}$ containing $X$ for PT method.

the closest B-scans and the probe trajectory at point $X$. An option would be to compute numerically this trajectory using a Runge-Kutta method. However, this would be computationally prohibitive. This problem is solved by computing the "virtual" plane that passes through $X$ in the sense of the probe trajectory (see Fig 10. For this purpose, we need first to compute the plane position in the 3D space. This can be achieved by determining the probe position relative to this "virtual" plane and then perform a rigid transformation to map from receiver to transmitter coordinates. This transformation is based on six parameters position (3 translations and 3 rotations) that allow a perfect localization of the plane in the $3 \mathrm{D}$ space. The tracking of the probe motion over time provides six different signals that corresponds of the variation of the position parameter with time. Thus, computing the position of the "virtual" plane amounts to computing its acquisition time. Let us denote $\pi_{t}$ this plane, two steps are necessary to determine the coordinates of $X$ in $\pi_{t}$. Firstly, the time stamp of $\pi_{t}$ must be evaluated. Secondly, this time $t$ is used to estimate the probe position at this moment.

Under the assumption that the probe speed is constant between two consecutive B-scans, the latency ratio is supposed to be equal to the distance ratio:

$$
t=\frac{d_{t i+1}}{d_{t i}+d_{t i+1}}\left(t_{i}\right)+\frac{d_{t i}}{d_{t i}+d_{t i+1}}\left(t_{i}+1\right)
$$

where $d_{t i}$ is the distance (in the sense of orthogonal projection) between the current voxel and the B-scan of time stamp $t_{i}\left(d_{t i}=\left\|X-X_{t i}^{D W}\right\|\right)$.

Once the time stamp of the "virtual" plane is computed, the probe position can be interpolated. The second step is a cubic interpolation of position parameters at time stamp $t$. The Key function is used to carry out a direct cubic interpolation and is defined as:

$$
\varphi(t)= \begin{cases}(a+2)|t|^{3}-(a+3) t^{2}+1 & \text { if } 0 \leq|t|<1, \\ a|t|^{3}-5 a t^{2}+8 a|t|-4 a & \text { if } 1 \leq|t|<2, \\ 0 & \text { if } 2 \leq|t|\end{cases}
$$


With $a=-\frac{1}{2}, \varphi$ is a $C^{1}$ function and a third order interpolation is obtained [7]. In practice, four B-scans are used for cubic interpolation. This seems an optimal trade-off between computational time and reconstruction quality. For example, the interpolation of rotation parameter along $x$ axis $R_{x}$ reads as:

$$
R_{x}(t)=\sum_{k=t_{i}-1}^{t_{i}+2} R_{x}(k) \varphi(t-k)
$$

The "virtual" plane certainly not contains $X$ numerically, despite the distance is infinitesimal. Therefore, $X_{t}$ is used as the projection of $X$ on the "virtual" plane (see Fig 1). Then, $X_{t i}^{P T}$ and $X_{t i+1}^{P T}$ are obtained directly, since they have the same $2 \mathrm{D}$ coordinates (defined in each B-scans) as $X_{t}$.

\section{Results}

\subsection{Material}

An Sonosite system with a cranial $7-4 M H z$ probe was used to acquire the ultrasound images. The positions of the B-scans was given by a magnetic miniBIRD system (Ascension Technology Corporation) mounted on the US probe. The StradX software [3] was used to acquire images and $3 D$ position data. A CIRS, Inc $13 D$ ultrasound calibration phantom was used. The phantom contains two calibrated volumetric ellipsoids test objects. At the acquisition depth, only one of the ellipsoids is in the field of view. The two sequences used for the experiments are composed of $510 \times 441$ B-scans (204 B-scans for fan motion and 222 B-scans for translation motion, see Fig. 2).
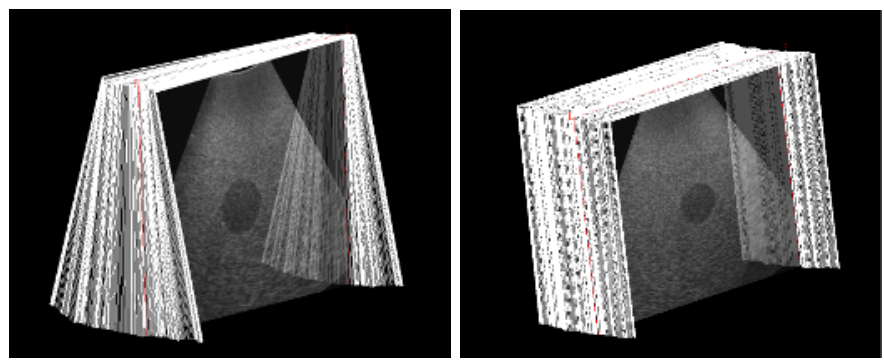

Fig. 2. B-scans sequences used during evaluation. Left: fan sequence. Right translation sequence.

\subsection{Evaluation Framework}

In order to perform an objective evaluation, the performance of the proposed method was compared with two other interpolation approaches: the voxel nearest neighbor (VNN) technique used in StackX [3] and the distance-weighted

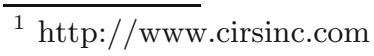


(DW) interpolation method presented in [8]. For the VNN method, each voxel is projected on the nearest B-scan and its luminance interpolated bilinearly. In the DW interpolation technique, each voxel is projected orthogonally on the $2 n$ nearest B-scans and its luminance interpolated (see section 2.1 and Fig. 11). To assess the reconstruction quality, evaluation data can be created from any image sequence: given a sequence of 3D freehand US, each B-scan is removed from the sequence and then reconstructed using the other B-scans of the sequence. This technique, "leaves one out", is performed in turn for each B-scan. Within this evaluation framework, the influence of two parameters is studied:

- "External" parameter associated with the acquisition setup: the acquisition frame rate. The sequence is sub-sampled thanks to SelectSX 2 , which simulates a lower frame acquisition rate. The sub-sampling factor varies from 2 to 6 , this means that we keep from one B-scan out of two (denoted by $1 / 2$ ) to one B-scan out of six (denoted by $1 / 6$ ).

- "Internal" parameter associated with the reconstruction method: the interpolation degree. The removed B-scans are reconstructed with different methods and different interpolation degree (from 1 to 2 for DW interpolation and PT method).

The Mean Square Error (MSE) is used as quality criterion:

$$
M S E(t)=\frac{1}{P} \sum_{j=1}^{P}\left(\tilde{I}_{t}\left(x_{j}\right)-I_{t}\left(x_{j}\right)\right)^{2}
$$

where $I_{t}$ is the original $t$ image (removed from the sequence), $\tilde{I}_{t}$ the reconstructed image and $P$ is the number of pixel in this B-scan. From $M S E$ estimation for all B-scans of the sequence, we compute the mean $\mu$ and the standard deviation $\sigma$ of reconstruction error.

$$
\begin{gathered}
\mu=\frac{1}{N-2} \sum_{t=2}^{N-1} M S E(t) \\
\sigma^{2}=\frac{1}{N-2} \sum_{t=2}^{N-1}(M S E(t)-\mu)^{2}
\end{gathered}
$$

$N$ is the total number of B-scan in the sequence. The first and last B-scans are rejected to avoid artifacts.

\subsection{Experiments}

Results are presented in Figure 3 and Table 1 Figure 3 shows the sub-sampling influence on reconstruction error relatively to motion nature (i.e. translation or fan), for interpolation degree equals to 1 . In all cases, the Probe Trajectory

$\overline{2}$ http://mi.eng.cam.ac.uk/ ${ }^{\sim}$ rwp/stradx/utilities.html 

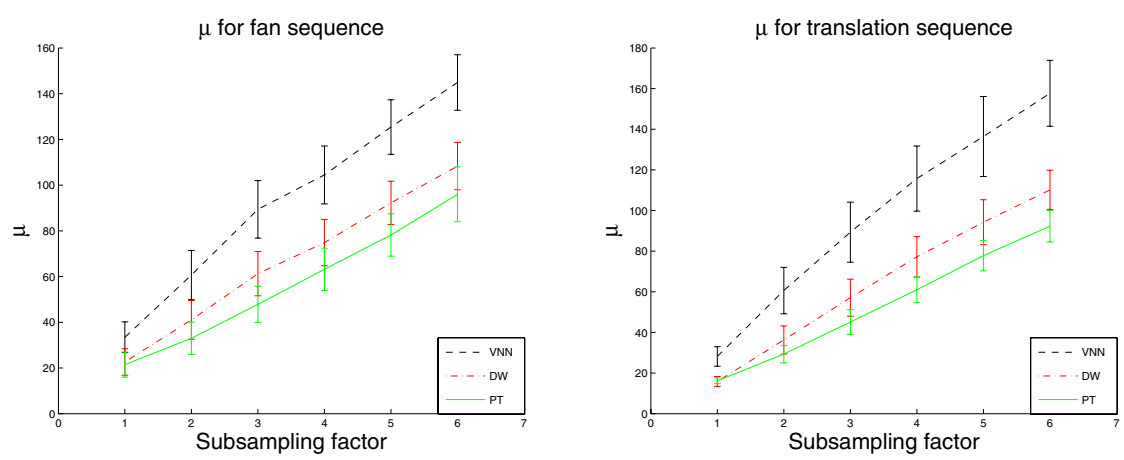

Fig. 3. Variation of error reconstruction relatively to sub-sampling factor with interpolation degree $=1$. Left fan motion. Right translation motion. Three methods are evaluated : VNN, DW and PT. The PT method outperforms others methods especially on sparse data.

Table 1. Error measures composed of mean $\mu$ and standard deviation $\sigma$ for the different methods. $L_{\text {time }}$ is the time spent for labeling, while $I_{\text {time }}$ is the time spent for interpolation. Error measures indicate that the PT method obtains better results than the VNN and DW methods. The improvement in terms of reconstruction quality is obtained at the expense of a slight computational increase.

\begin{tabular}{|l|l|ccc|ccc|ccc|}
\hline Sequence & Order & \multicolumn{3}{|c|}{ VNN } & \multicolumn{3}{|c|}{ DW } & \multicolumn{3}{c|}{ PT } \\
& & $\mu$ & $\sigma$ & $I_{\text {time }}$ & $\mu$ & $\sigma$ & $I_{\text {time }}$ & $\mu$ & $\sigma$ & $I_{\text {time }}$ \\
\hline fan & $n=1$ & 33.4 & 13.5 & $20 s$ & 22.6 & 11.6 & $44 s$ & 21.5 & 11.0 & $114 s$ \\
$L_{\text {time }}=414 s$ & $n=2$ & & & & 26.2 & 12.5 & $46 s$ & 22.9 & 10.6 & $122 s$ \\
fan $1 / 2$ & $n=1$ & 60.7 & 21.6 & $21 s$ & 41.1 & 16.9 & $31 s$ & 33.0 & 14.2 & $114 s$ \\
$L_{\text {time }}=215 s$ & $n=2$ & & & & 46.6 & 17.6 & $44 s$ & 38.2 & 14.1 & $124 s$ \\
fan $1 / 3$ & $n=1$ & 89.4 & 25.2 & $20 s$ & 61.3 & 19.4 & $30 s$ & 47.8 & 15.9 & $111 s$ \\
$L_{\text {time }}=145 s$ & $n=2$ & & & & 67.6 & 18.6 & $43 s$ & 56.2 & 14.2 & $118 s$ \\
\hline translation & $n=1$ & 28.2 & 9.6 & $20 s$ & 15.9 & 4.8 & $37 s$ & 16.2 & 2.9 & $138 s$ \\
$L_{\text {time }}=42 s$ & $n=2$ & & & & 19.1 & 6.4 & $49 s$ & 18.3 & 3.5 & $149 s$ \\
translation $1 / 2$ & $n=1$ & 60.6 & 22.9 & $21 s$ & 36.3 & 13.9 & $32 s$ & 29.3 & 8.6 & $138 s$ \\
$L_{\text {time }}=27 s$ & $n=2$ & & & & 43.0 & 14.5 & $47 s$ & 35.8 & 8.7 & $147 s$ \\
translation $1 / 3$ & $n=1$ & 89.3 & 29.6 & $20 s$ & 57.1 & 18.3 & $31 s$ & 45.1 & 12.1 & $138 s$ \\
$L_{\text {time }}=20 s$ & $n=2$ & & & & 63.7 & 17.5 & $46 s$ & 52.9 & 11.1 & $146 s$ \\
\hline
\end{tabular}

(PT) method outperforms VNN and DW methods especially on sub-sampled sequences. On sparse data sets, the incorporation of probe trajectory leads to a more robust reconstruction. In Table 1] error measures and computation time are presented. Reconstructions were performed on P4 3.2 Ghz with 2Go RAM. In order to only compare interpolation times, the total computational time was split in two : $L_{\text {time }}$ corresponds to labeling step and second denoted by $I_{\text {time }}$ is interpolation time. The labeling step consist in $K_{n}$ construction for each 
voxel $X$. Our implementation of methods can be largely optimized, as compared to StackSX (22s for all reconstruction process within VNN method). Although the labeling step need significant improvement, this study aims to compare computation time between identical implementations. Increase in quality for PT method is obtained at the expense of slight computation time increase. Nonetheless, this side effect is reasonable with regards to quality reconstruction. Contrary to more elaborated techniques like non-rigid registration or RBF, which are considerably computationally expensive, the PT approach offers an attractive compromise between time computation and quality reconstruction. Figure 4 shows the differences between original and reconstructed B-scan with the VNN, DW and PT methods. Visually, the PT reconstruction appears closer to the original B-scan, which is in agreement with the numerical results of Table 1. Reconstruction results on real dataset are presented in Figure 5, The VNN method leads to a lot of discontinuities. The differences between the DW and

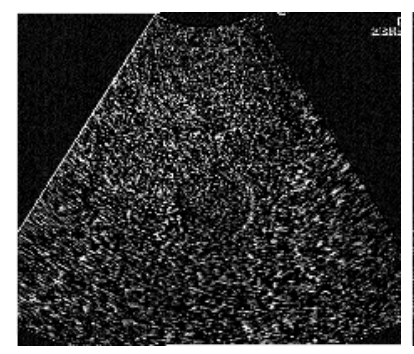

VNN

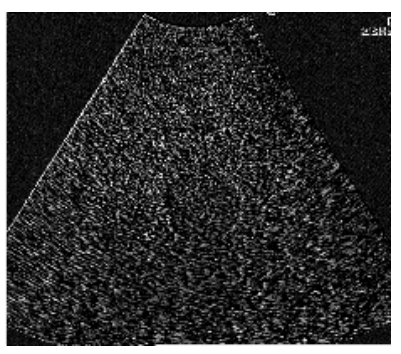

DW

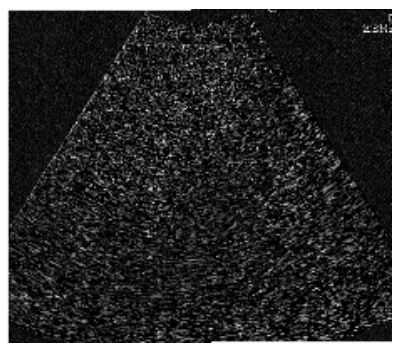

PT

Fig. 4. Differences between original and reconstructed B-scan for not subsampled fan sequence. From left to right VNN, DW and PT methods. This shows that error between reconstructed B-scan and "ground truth" is visually better with the PT method.

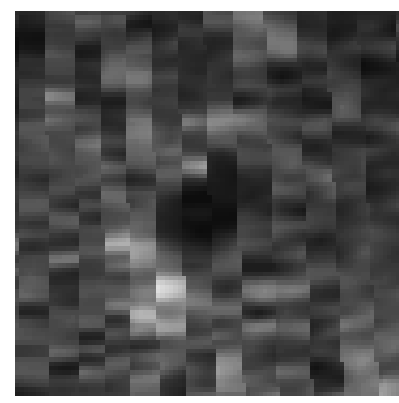

VNN

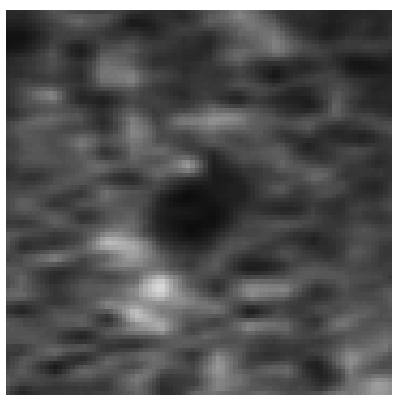

DW

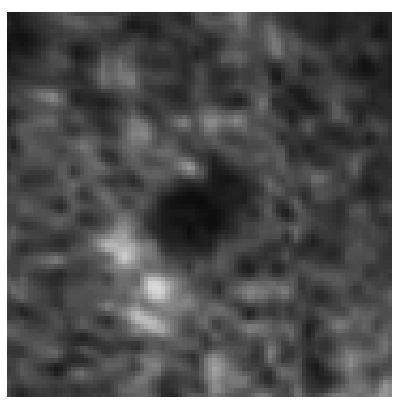

PT

Fig. 5. Zoom on hepatic vessels extracted from liver reconstruction with degree $=1$ and subsampling factor $=4$. From left to right the VNN, DW and PT methods. The images are extracted from $3 \mathrm{D}$ volume along the temporal axis $(\mathrm{z})$ in order to under-light inherent artifacts of the VNN (i.e. discontinuities) and the DW (i.e. blur) methods. The PT method is more efficient at preserving the native texture pattern of US image than the DW method. 
PT methods are less visible, as assessed by numerical results. Nevertheless, in general the DW method smooths out the edges and spoils the native texture pattern of US image more than the PT method (see Fig. 5 ).

\section{Conclusion}

This paper presented a 3D freehand US reconstruction method using probe trajectory information. This method performs better than traditional reconstruction approaches with a reasonable increase in computation time. Results on sub-sampled acquisitions show that the probe trajectory brings a relevant information in the reconstruction process. The main limitation of PT method is the assumption of constant probe speed between two slices. Nevertheless, for frame rate around $5-15 \mathrm{~Hz}$ and moderate care during acquisition (i.e. a relatively continuous motion user) this limitation can be easily overcome. Further work should pursue the comparison between the PT reconstruction approach and registration-based approaches [2. Finally, our implementation should be largely optimized using graphic library implementation (ex : Open $G L$ ) or even GPU one.

\section{References}

1. C. D. Barry, C. P. Allott, N. W. John, P. M. Mellor, P. A. Arundel, D. S. Thomson, and J. C. Waterton. Three dimensional freehand ultrasound: image reconstruction and volume analysis. Ultrasound in medecine and biology, 23(8):1209-1224, 1997.

2. G. P. Penney, J. A. Schnabel, D. Rueckert, M. A. Viergever, and W. J. Niessen. Registration-based interpolation. IEEE Trans Med Imaging, 23(7):922-926, 2004.

3. R. W. Prager, A. H. Gee, and L. Berman. Stradx : real-time acquisition and visualization of freehand three-dimensional ultrasound. Medical Image Analysis, 3(2):129140, 1999.

4. R. Rohling, A. H. Gee, L. H. Berman, and G. M. Treece. Radial basis function interpolation for freehand 3D ultrasound. In Attila Kuba, Martin Sámal, and Andrew Todd-Pokropek, editors, Information Processing in Medical Imaging, volume 1613 of $L N C S$, pages 478-483. Springer, 1999.

5. J. M. Sanchez and J. S. Marques. A rayleigh reconstruction/interpolation algorithm for 3D ultrasound. Pattern recognition letters, 21:917-926, 2000.

6. S. Sherebrin, A. Fenster, R. N. Rankin, and D. Spence. Freehand three-dimensional ultrasound: implementation and applications. In Proc. SPIE Vol. 2708, p. 296-303, Medical Imaging 1996: Physics of Medical Imaging, Richard L. Van Metter; Jacob Beutel; Eds., pages 296-303, April 1996.

7. P. Thévenaz, T. Blu, and M. Unser. Interpolation revisited. IEEE Transactions on Medical Imaging, 19(7):739-758, 2000.

8. J. W. Trobaugh, D. J. Trobaugh, and W. D. Richard. Three dimensional imaging with stereotactic ultrasonography. Computerized Medical Imaging and Graphics, 18(5):315-323, 1994. 